\title{
Hyper suprime-cam for weak gravitational lensing survey (Presentation Video)
}

\section{Satoshi Miyazaki}

Satoshi Miyazaki, "Hyper suprime-cam for weak gravitational lensing survey (Presentation Video)," Proc. SPIE 9143, Space Telescopes and Instrumentation 2014: Optical, Infrared, and Millimeter Wave, $91431 Z$ (22 June 2014); doi: 10.1117/12.2063486

EDent: SPIE Astronomical Telescopes + Instrumentation, 2014, Montréal, Quebec, Canada 


\title{
Hyper Suprime-Cam for Weak Gravitational Lensing Survey (Presentation Video)
}

\author{
Satoshi Miyazaki, National Astronomical Observatory of Japan (Japan)
}

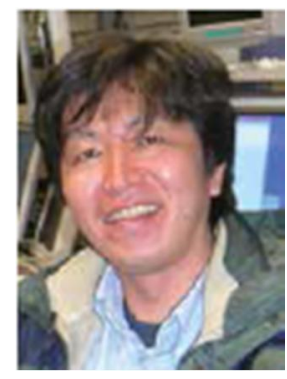

\begin{abstract}
Hyper Suprime-Cam (HSC) is a next generation wide field optical imaging camera built for $8.2 \mathrm{~m}$ Subaru telescope. The field of view is 1.5 degree in diameter and the nearly $50 \mathrm{~cm}$ image circle was paved by 116 fully depleted CCDs (2k x $4 \mathrm{k}$ 15 micron square pixels). To realize a seeing limit imaging at Mauna Kea, the specification on the overall instrument PSF is set as 0.32 arc-second (FWHM). This is crucial for our primary scientific objectives: weak gravitational weak lensing survey to probe dark matter distribution. We started building the camera in 2006, had a first light in 2012 and now in the final phase of the commissioning. The delivered image quality is mostly seeing limit as designed and we once observed the seeing size of 0.43 arc-second (median value over the field of view) in Y-band with 300 seconds exposure. Our 300 nights observing proposal has been already accepted. The program starts in March 2014 and continues over 5 years.
\end{abstract}

View presentation video on SPIE's Digital Library: http://dx.doi.org/10.1117/12.2063486.3664789960001 